\title{
Correction to: Effects of metaperiodate and urea solutions on the serological diagnosis of human sporotrichosis using an indirect ELISA test
}

\author{
Letícia Maria Leomil Coelho $^{1}$ - Julianne Caravita Grisolia ${ }^{1}$ - Maria Lúcia ${ }^{2}$ - Míriam Boczar ${ }^{2}$ - Eric Batista Ferreira ${ }^{3}$. \\ Denismar Alves Nogueira ${ }^{3}$. Jorge Kleber Chavasco ${ }^{1}$ • Zoilo Pires de Camargo ${ }^{4}$ - Leila Maria Lopes-Bezerra ${ }^{5}$. \\ Luiz Felipe Leomil Coelho ${ }^{1}$. Luiz Cosme Cotta Malaquias ${ }^{1}$
}

Published online: 14 March 2019

(C) Sociedade Brasileira de Microbiologia 2019

Correction to: Brazilian Journal of Microbiology (2019) 50:139-145 https://doi.org/10.1007/s42770-018-0005-6

In the article mentioned above an author's name was misspelled.

The correct author name reads as follows:

Leila Maria Lopes-Bezerra. We apologize for the inconvenience.

Publisher's note Springer Nature remains neutral with regard to jurisdictional claims in published maps and institutional affiliations.

The online version of the original article can be found at https://doi.org/ 10.1007/s42770-018-0005-6

Luiz Cosme Cotta Malaquias

luizcosme@hotmail.com

1 Departamento de Microbiologia e Imunologia, Instituto de Ciências Biomédicas, Universidade Federal de Alfenas, Rua Gabriel Monteiro da Silva, 700 - Centro, Alfenas, MG CEP 37130-001, Brazil

2 Universidade José do Rosário Vellano, Alfenas, MG, Brazil

3 Instituto de Ciências Exatas, Universidade Federal de Alfenas, Alfenas, MG, Brazil

4 Departamento de Microbiologia, Imunologia e Parasitologia, Universidade Federal de São Paulo, São Paulo, SP, Brazil

5 Laboratório de Micologia Celular e Proteômica, Universidade do Estado do Rio de Janeiro, Rio de Janeiro, RJ, Brazil 